\title{
A Survey on Information Diffusion in Online Social Networks
}

\author{
Yang Yujie \\ Department of Engineering, Wuhan University, China \\ 496582771@qq.com
}

\begin{abstract}
Nowadays, social networks have become a critical data dissemination platform with the drastic proliferation of social networks and the growing recipient of data. In this paper, recent successful works of information diffusion in online social networks are introduced. We summarize several classic information diffusion models like an explanatory model: the SI Model, the SIS Model, the SIRS Model; predictive Model: Independent Cascade Model, Linear Threshold Model. Then, we discuss some applications of those information diffusion model in different social networks.
\end{abstract}

\section{CCS Concepts}

-Applied computing $\rightarrow$ Operations research $\rightarrow$ Industry and manufacturing $\rightarrow$ Supply chain management

\section{Keywords}

Information diffusion; Online social networks; Disease model; Cascade model.

\section{INTRODUCTION}

Social networks have become a critical data dissemination platform with the drastic proliferation of social networks and the growing recipient of data. Users also experience the adverse effects of damaging data when exchanging data. Research on the dissemination of data in social networks online is, therefore, worthwhile.

Information dissemination is an extensive field of research and has been attractive in many areas, such as physics, biology, etc. The spread of technology over a network and population disease spray has been researched for thousands of years, one of the original variables for the study of networks.

Some researchers in the field of information diffusion have suggested several methods. [1] Compare the methods, algorithms, or techniques of spreader detection. [2] Talk about the information diffusion parameters. These parameters include bond strength, homophobia, groups, pictures, user roles, and

Permission to make digital or hard copies of all or part of this work for personal or classroom use is granted without fee provided that copies are not made or distributed for profit or commercial advantage and that copies bear this notice and the full citation on the first page. Copyrights for components of this work owned by others than ACM must be honored. Abstracting with credit is permitted. To copy otherwise, or republish, to post on servers or to redistribute to lists, requires prior specific permission and/or a fee. Request permissions from Permissions@acm.org.

ESSE 2020, November 6-8, 2020, Rome, Italy

(C) 2020 Association for Computing Machinery.

ACM ISBN 978-1-4503-7762-1/20/11 ...

https://doi.org/10.1145/3393822.3432322 topics. Dey et al. [3] are studying the related topic evaluation references, dissemination of information, and the features of social ties in the context of online social networks. Dong et al. [4] divided existing models on the basis of studies, some of them from a true social network and some of those not into theoretical dissemination models and waterfall models. Rumor is one of the significant manifestations of online social networks dissemination of data. One of the current techniques of managing the dissemination of data is to use connection prediction, disseminate data through current rumors, predict further dissemination of rumors and cut off possible connections of transmission to accomplish the objective of weakening rumors. Another efficient technique is to discover the source of the rumor [5,6,7], which is of excellent assistance in managing the spread of rumors efficiently.

A typical technique is to find the source point based on the network propagation snapshot for the place of the source issue. For example, [8] suggested a location technique for the sensitive virus propagation model based on the minimum description length, which can automatically determine the number of source points of propagation and recognize various source nodes of diffusion in the networks.

Some scientists suggested techniques for identifying sources of diffusion in networks. These techniques concentrate primarily on identifying a single source of diffusion in tree networks. Zhu and Ying [9] have proposed a method for the detection of tree network diffusion sources using a sample path-based method with a snapshot observation.

The remainder of this document is as follows, structured. In Section 2, we describe in detail some conventional models of information diffusion. In Section 3, we deal with some communications model applications in different social networks. In the last section, we summarize the methods examined in taxonomy.

\section{INFORMATION DIFFUSION MODEL 2.1 Explanatory Model}

\subsubsection{The SI Model}

The SI is the purest type of all model epidemics. Individuals do not have immunity during the simulation. They are born. After being infected and without therapy, people remain infected and infectious and maintain in touch with vulnerable persons throughout their lifetimes. Some scientists have implemented and achieved certain results in applying the SI model to the theory of data transmission.

The SI model is the cleanest form of all illness patterns. In the simulation, individuals were born without immunity. Once infected, individuals stay in contact with a susceptible 
population, remaining infected, and infectious throughout their life. Some researchers have applied the SI model to information theory and have achieved certain achievements.

J Jiang et al. [10] suggested a new technique for identifying various sources of diffusion related to the probability of propagation and the number of hops between nodes. To show the diffusion dynamics feature of each node, they embraced the classic susceptible-infected model. Prakash, B. Aditya et al. [11] suggested that the Minimum Description Length (MDL) principle be used to define that group of seed nodes and that virus spread ripple from those nodes that best describe the snapshot provided. First, discovering high-value seeds because of the number of plants and then taking our thoroughly constructed MDL rating to identify the real amount of plants.

Mei, Ren-Jie et al. [12] suggested some ideas that the impacts of social strengthening usually inhibit the propagation of data while at the same time contributing to the creation of certain hot spots. Effects of social strengthening prevent the spread of data, although it usually contributes simultaneously to the creation of some hot spots. The main goal was to find the method that performs on large complex networks in a reasonable time and at the same time, delivers high-quality localization results. Paluch, Robert, et al. [13] suggest a new strategy that ignores observers with low-quality data and selects prospective sources based on the probability gradient of high-quality observers.

\subsubsection{The SIS Model}

The SIS Epidemic Model is a way to model illnesses by classifying the population on the basis of their condition. These kinds of models are generally referred to as SIR models, referring to Susceptible, Infected, and Removed / Recovered as the three status of the disease in which the population may fall. Based on these categories, we can see that the SIS model is one that models illnesses that do not give immunity to the disease to recovered people and are again prone to infection after recovery. The SIS model can be used to define sexually transmitted diseases such as syphilis or gonorrhea in which people are treated and recovered but are immediately again vulnerable.

It is not practical because of the SI model, as it does not allow infected users to be healed after infection. This problem is addressed by the SIS model. Newman [14,15] and Gross et al. [16] investigated complicated and adaptive networks. In the field of Information Diffusion, his sort of disease model can also be implemented. Lin, Yishi, et al. [17] correctly predict diffusion behavior, design a big network of easy and efficient vaccination or advertising strategy. They allow intermediate states to exist between sensitive and infected states.

\subsubsection{The SIRS Model}

The SIR model assumes that people suffer from lifelong immunity diseases once they have recovered. It is the case for a number of diseases. For example, the immunity of a person may decrease over time due to seasonal flu, another class of airborne disease. In this scenario, the SIRS model is implemented to render the population recovered vulnerable.

Chen et al. [18] examine the identification of different information sources in networks within the Susceptible Infected Recovered Model, assuming the information sources quality is known, creating a sample path algorithm called trees and place clusters. The estimators generated by the suggested algorithm for periodic trees are at a steady distance with a high probability from the correct sources. In addition, present a general network heuristic algorithm and an algorithm to estimate the number of sources when the amount of actual sources is unknown.

Zhu, Kai, et al. [19] investigated the question of finding the information source in a network following the common information distribution process. Recovered Susceptible Infected model assumes that each network node is initially in a susceptible position other than the information source in the infected scenario. Susceptible people may then be infected by infected persons, and infected persons will recover and will not be infected again after regeneration. They generate a trajectory-based sample approach where the information source estimator is chosen as the root node linked to the sample route, which most likely helps to find the origin of the snapshot in light of the snapshot and network topology.

\subsection{Predictive Model}

Kether et al. [20] proposed that, in accordance with prior works on social network analysis, interactive particulate system and marketing, two main influencing models, an autonomic cascades (IC) model, and a linear threshold (LT) model. A social network is modeled on the guided graph $\mathrm{G}=(\mathrm{V}, \mathrm{E})$ in both models, in which V's cervixes represent relationships with individuals and E's edges, and edge orientations mean an influence direction. Each edge in the IC model has an activation probability and effect is transferred by activated nodes which independently trigger their inactive neighbors in the function of their edge activation probabilities. Each edge of the LT model has a weight, every vertex a randomly selected threshold and a vertex is enabled if the threshold of its active neighbors is exceeded by a weighted amount.

\subsubsection{Independent Cascade Model}

The Independent Cascade Model (IC) and Linear Threshold Model (LT) are the two classic techniques for portraying the spread of impact in online social networks, respectively explaining the spread from the perspective of probability and threshold. Since their suggestion, both techniques have been commonly quoted and developed.

Kempe et al. [20] initially proposed the issue of maximizing impact and suggested two fundamental models of diffusion impact-the Independent Cascade (IC) model and the Linear Threshold (LT) model. Yang, Wenjing et al. [21] suggested an IC(t-IC) model that would take time into consideration. There are two important components of impact diffusion in the real-world time constraint and continuing activation tests which lack current impact diffusion models and effective processing algorithms. The t-IC model incorporates these two elements in its mechanism of activation in order to make their impact more realistic in social networks.

In[ 22], which takes into account the diffusion of negative and positive opinions, an IC model with negative opinions (IC-N) is recommended. In this paper, they propose an extension of the independent cascade model to the evolution and dissemination of negative opinions. The new model has an explicit quality factor in the model of natural conduct of individuals because of product defects that turn a product into a negative one. They also design an effective algorithm for calculating the impact on tree structures, which is nontrivial because of the model's negative bias. The developers use this algorithm as the heart for the construction of a heuristic algorithm to maximize the impact of general graphs. 
Saito, Kazumi et al. [23] tackle the issue of anticipating the probability of diffusion in complex networks and focusing on the autonomous cascade (IC) model and defining the probability of data diffusion episodes where an episode is a series of freshly engaged nodes. Then, using the EM algorithm, they present a technique for anticipating the probabilities of diffusion. Finally, the suggested technique operates well with the use of a true network information set.

\subsubsection{Linear Threshold Model}

The linear threshold model was first suggested to define collective behavior[1] and was implemented in economics and sociology to describe a sequence of binary decision events.

The research by Chen $\mathrm{W}$ et al. [24] influences maximization under the linear threshold model; one of the significant models is to formalize the conduct of propagating impact on social networks. They proposed the first scalable heuristic algorithm, which is based on a fast effect calculation for DAGs, tailored to maximize the effect on the LT model, known as the LDA G algorithm. They conduct a comprehensive simulation, showing that their algorithm can be scaled to a system with millions of nodes and edges so as to reach a magnitude faster than the greedy approximative algorithm suggested by Kămp et al.[ 20], and their optimized variants. and works continually amongst the best algorithms.

Pathak et al. [25] have a generalized model of the linear limit to simulate different network waterfalls, allowing people to switch between them. The model proposed is a rapid blending of the Markov chain, and the corresponding stable distribution is used to estimate the highly likely distribution position of the waterfalls in the network. The results demonstrate the elevated value of the solution estimated on several real-world networks. The generalized linear threshold model, together with an algorithm, is being introduced by Zhang, Huiyuan et al. [26] to estimate the most likely spread of cascades in a specific network. Outputs from a variety of applications on actual global networks are studied with the proposed algorithm.

Impact Maximization is the problem of selecting a small number of seed nodes in a social network to maximize their overall effect coverage under particular diffusion effect modeling. In the model of the competitive linear threshold (CLT) He, Xinran, et al. [27] examined the spread of the competitive effect of the social networks to extend the classical linear threshold model. The CLT model concentrates on the problem that an organization tries, by selecting an amount of seed knots strategically, to block its rivals ' effect as much as possible, by calling it a problem of blocking maximization (IBM). Because of this issue, the CLT model focuses on the problem.

The fresh propagation model, Litou, Iuliana, etc. [28] proposed, namely Dynamic Linear Threshold (DLT), that effectively reflects how conflicting information, i.e., misinformation and trustworthy information, propagate via the network. They formulate an issue of optimization based on the DLT model to define the best subset of customers to prevent misinformation spread by spreading reliable information.

\section{APPLICATION OF COMMUNICATION MODEL IN DIFFERENT SOCIAL NETWORKS}

Budak et al. [29] use the Independent Cascade Model to study the spreading procession in social networks of competing campaigns. They discovered the best way to disseminate "excellent data" in the presence of a misinformation cascade, which will minimize the catastrophic impacts of the misinformation campaign. To restrict the spread of misinformation, they study the "bad" nodes in the networks. For example, they are looking for ways to ensure that most social network users hear about the right information in front of the wrong one. You can also apply the techniques in this article to any two competing campaigns that spread concurrently across the network.

Zang et al. [30] present, and evaluate their distinctions, three propagation models (SI, IC, and SIR models). They present a multi-source location technique based on a specified snapshot of infected nodes in the network that are partly and sparsely observed. In addition, then use community cluster algorithms to alter the issue of multi-source location into a lot of single sources locating issues. They recognize the nodes with the most significant estimates of probability as to the source node on the infected clusters at the last step.

Comin et al. [31] evaluated three propagation spreads: snowball (also known as dilation), diffusion, and contact process based on the susceptible-infected (SI) template, then proposed and validated an efficient methodology to identify the source nodes. The method is based on calculating the centrality of the nodes on the sampled network, expressed here by degree, betweenness, proximity, and centrality of the convector. It is shown that the source node tends to have the most significant values of measurement.

Jain et al. [33] assume that rumor spreads across the network in a disciplined susceptible-infected (SI) model to capture the dynamism of rumor propagation. They think in this article that it is of enormous significance to rapidly-produce rumor source estimates without seriously compromising on precision. Based on the hitting time statistics of a surrogate random walk method, they developed a heuristic for this, which can be used to approximate the MLE of the rumor source to infer the rumor source.

Luo et al. [33] address the problem of estimating an infection source for an IS model that does not include all infected nodes. In this paper, they include a discrete IS model which describes how an infection spreads to find only a limited amount of information on the source of infection. In addition, if not all infected nodes can be observed, estimators of infection sources for the SI model have been acquired. They showed that when the network is a tree, the estimator is a Jordan center. They proposed an efficient $\mathrm{O}(\mathrm{n})$ complexity algorithm to find the estimator of the network size where $n$ is. For specific networks, the sources estimated based on a highly complex MIQCQP formula and a complex $\mathrm{O}(\mathrm{n} 3)$ heuristic algorithm have been suggested.

Dong et al. [34] studies a single source problem for evaluating the IS model with a special a priori knowledge of the suspect knot set; they consider the question of the rely on the snapshot observation of infected knots in a network for a certain rumor among a number of suspected individuals.

The problem for the Susceptible Infected-Recovers (SIR) model, which is the one-source evaluation, Zhu et al. [35] considered, can recover but never be infected again by the infected node. Due to a network snapshot, which understands all infected nodes but can not distinguish between susceptible nodes and 
nodes, they try to detect the information source based on the topology and network snapshots. It developed an example path-based strategy where the root node linked to the sample path was selected for the data source estimator, most likely contributing to the snapshot observed, and showed that the sample path-based estimator is a node with minimal eccentricities of infection.
In a research of the Susceptible-Infected-Susceptible model, Luo et al. [33] study a one source estimate where the node found is likely to be reinfected. At some point, they assume they observe only one snapshot of the infection spreading technique and obtain a calculator that identifies the source node linked to the most likely infection technique producing the snapshot.

Table 1. The summary of different researches

\begin{tabular}{|c|c|c|c|c|}
\hline Paper & Method & Evaluation & Measure & Result \\
\hline [10] & K-center & $\begin{array}{l}\text { the North American Power } \\
\text { Grid; } \\
\text { the Yeast protein-protein } \\
\text { interaction network; the } \\
\text { Facebook network. }\end{array}$ & $\begin{array}{l}\text { Effective } \\
\text { distance }\end{array}$ & $\begin{array}{l}\text { identifying the diffusion sources and estimating the spreading } \\
\text { time.More than } 80 \% \text { of experiment runs can accurately estimate the } \\
\text { number of sources when }|\mathrm{S} *|=2 \text {, and around } 60 \% \text { when }|\mathrm{S} *|=3 \text {. }\end{array}$ \\
\hline$[13]$ & GMLA & $\begin{array}{l}\text { Erdös-Rényi, Barabási-Albert } \\
\text { networks and performed on } \\
\text { Gnutella. }\end{array}$ & $\begin{array}{l}\text { Propagation } \\
\text { ratio }\end{array}$ & $\begin{array}{l}\text { identifying the diffusion source based on the snapshot of an entire } \\
\text { network at a certain time instance, or a small subset of nodes all the } \\
\text { time. }\end{array}$ \\
\hline$[19]$ & $\begin{array}{l}\text { Reverse } \\
\text { Infection }\end{array}$ & Tree networks. & $\begin{array}{l}\text { The sample } \\
\text { path. }\end{array}$ & $\begin{array}{l}\text { finding the information source based on the snapshot. The detection } \\
\text { rate of the algorithm is higher than that of the closeness centrality } \\
\text { heuristic by around } 20 \% \text { when the degree is small and by } 10 \% \text { when } \\
\text { the degree is large }\end{array}$ \\
\hline
\end{tabular}

Table 2. The summary of different researches

\begin{tabular}{|c|c|c|c|c|}
\hline Paper & Method & Evaluation & Measure & Result \\
\hline$[23]$ & EM & $\begin{array}{l}\text { Exploited the blog } \\
\text { "Theme salon of } \\
\text { blogs" in the site } \\
\text { "goo" }\end{array}$ & $\begin{array}{l}\text { The likelihood for } \\
\text { multiple episodes }\end{array}$ & Predicting diffusion probabilities in complex networks \\
\hline$[24]$ & LDAG & $\begin{array}{l}\text { NetHEPT; DBLP the } \\
\text { Computer Science } \\
\text { Bibliography } \\
\text { Database maintained } \\
\text { by Michael Ley; } \\
\text { Epinions ; Amazon } \\
\text { dated on March 2, } \\
2003\end{array}$ & $\begin{array}{l}\text { The scalability of the } \\
\text { algorithms }\end{array}$ & $\begin{array}{c}\text { Finding a small set of most influential nodes in a social } \\
\text { network so that their aggregated influence in the } \\
\text { network is maximized }\end{array}$ \\
\hline$[25]$ & StochColor & Methodology & $\begin{array}{l}\text { The number of cascades' } \\
\text { surviving }\end{array}$ & $\begin{array}{l}\text { Discovering the most likley states of the cascades' } \\
\text { spread in a given graph. }\end{array}$ \\
\hline
\end{tabular}




\begin{tabular}{|c|c|c|c|c|}
\hline [27] & CLDAG & $\begin{array}{c}\text { Mobile } \\
\text { network(derived from } \\
\text { a partial call detailed } \\
\text { record (CDR) data of } \\
\text { a Chinese city from } \\
\text { China Mobile ); } \\
\text { collaboration } \\
\text { networks(The } \\
\text { NetHEPT and } \\
\text { NetPHY ) }\end{array}$ & $\begin{array}{l}\text { Numerber } \\
\text { of positive } \\
\text { seeds and } \\
\text { running } \\
\text { time }\end{array}$ & $\begin{array}{l}\text { Selecting positive seed nodes. The negative influence } \\
\text { reduction of CLDAG is on average } 78.24 \% \text { higher than } \\
\text { that of the proximity algorithm. For max-degree } \\
\text { negative seed selection, CLDAG improves the } \\
\text { performance of proximity heuristic even more, for } \\
80.75 \% \text { on average. }\end{array}$ \\
\hline [29] & $\begin{array}{c}\text { Degree } \\
\text { centrality/Early } \\
\text { infectees/Largest } \\
\text { infectees. }\end{array}$ & $\begin{array}{l}\text { A number of } \\
\text { close-knit regional } \\
\text { networks obtained } \\
\text { from the Facebook } \\
\text { social network }\end{array}$ & & $\begin{array}{l}\text { Limiting the effects of misinformation in a social } \\
\text { network. }\end{array}$ \\
\hline
\end{tabular}

\section{CONCLUSION}

In this article, we examined several latest effective reports of online social networking dissemination of data. For the first time, we discussed some conventional models of information diffusion like an explanatory model: the SI Model, the SIS Model, the SIRS Model, the SIRS Model. Then we address some apps in various social networks of that data dissemination model. We can conclude from those literary works that such work is important and that future apps can promote government surveillance, marketing, and so on for decision making. This kind of studies is important.

\section{REFERENCE}

[1] Kumaran, P., and S. Chitrakala. "A survey on influence spreader identification in the online social network." 2016 International Conference on Information Communication and Embedded Systems (ICICES). IEEE, 2016.

[2] Wani, Mudasir, and Manzoor Ahmad. "Information diffusion modelling and social network parameters (A survey)." Proceedings of the International Conference on Advances in Computers, Communication and Electronic Engineering, Kashmir, India. 2015.

[3] Dey, Kuntal, Saroj Kaushik, and L. Venkata Subramaniam. "Literature survey on interplay of topics, information diffusion and connections on social networks." arXiv preprint arXiv:1706.00921 (2017).

[4] Li, Dong, et al. "A survey on information diffusion in online social networks." Chinese Journal of Computers 37.1 (2014): 189-206.

[5] Shah, Devavrat, and Tauhid Zaman. "Detecting sources of computer viruses in networks: theory and experiment." ACM SIGMETRICS Performance Evaluation Review. Vol. 38. No. 1. ACM, 2010.

[6] Brockmann, Dirk, and Dirk Helbing. "The hidden geometry of complex, network-driven contagion phenomena." science 342.6164 (2013): 1337-1342.

[7] Lokhov, Andrey Y., et al. "Inferring the origin of an epidemic with a dynamic message-passing algorithm." Physical Review E 90.1 (2014): 012801.
[8] Prakash, B. Aditya, Jilles Vreeken, and Christos Faloutsos. "Spotting culprits in epidemics: How many and which ones?." 2012 IEEE 12th International Conference on Data Mining. IEEE, 2012.

[9] Zhu, Kai, and Lei Ying. "Information source detection in the SIR model: A sample-path-based approach." IEEE/ACM Transactions on Networking (TON) 24.1 (2016): 408-421.

[10] Jiang, Jiaojiao, et al. "K-center: An approach on the multi-source identification of information diffusion." IEEE Transactions on Information Forensics and Security 10.12 (2015): 2616-2626.

[11] Prakash, B. Aditya, Jilles Vreeken, and Christos Faloutsos. "Spotting culprits in epidemics: How many and which ones?." 2012 IEEE 12th International Conference on Data Mining. IEEE, 2012.

[12] Mei, Ren-Jie, et al. "Modeling for heterogeneous multi-stage information propagation networks and maximizing information." Chinese Physics B 28.2 (2019): 028701 .

[13] Paluch, Robert, et al. "Fast and accurate detection of spread source in large complex networks." Scientific reports 8.1 (2018): 2508.

[14] Newman, Mark EJ. "The structure and function of complex networks." SIAM review 45.2 (2003): 167-256.

[15] Newman, Mark EJ. "Threshold effects for two pathogens spreading on a network." Physical review letters 95.10 (2005): 108701.

[16] Gross, Thilo, Carlos J. Dommar D’Lima, and Bernd Blasius. "Epidemic dynamics on an adaptive network." Physical review letters 96.20 (2006): 208701.

[17] Lin, Yishi, et al. "Modelling multi-state diffusion process in complex networks: theory and applications." Journal of Complex Networks 2.4 (2014): 431-459.

[18] Chen, Zhen, Kai Zhu, and Lei Ying. "Detecting multiple information sources in networks under the SIR model." IEEE Transactions on Network Science and Engineering 3.1 (2016): 17-31. 
[19] Zhu, Kai, and Lei Ying. "Information source detection in the SIR model: A sample-path-based approach." IEEE/ACM Transactions on Networking (TON) 24.1 (2016): 408-421.

[20] Kempe, David, Jon Kleinberg, and Éva Tardos. "Maximizing the spread of influence through a social network." Proceedings of the ninth ACM SIGKDD international conference on Knowledge discovery and data mining. ACM, 2003.

[21] Yang, Wenjing, Leonardo Brenner, and Alessandro Giua. "Influence Maximization in Independent Cascade Networks Based on Activation Probability Computation." IEEE Access 7 (2019): 13745-13757.

[22] Chen, Wei, et al. "Influence maximization in social networks when negative opinions may emerge and propagate." Proceedings of the 2011 siam international conference on data mining. Society for Industrial and Applied Mathematics, 2011.

[23] Saito, Kazumi, Ryohei Nakano, and Masahiro Kimura. "Prediction of information diffusion probabilities for independent cascade model." International conference on knowledge-based and intelligent information and engineering systems. Springer, Berlin, Heidelberg, 2008.

[24] Chen W , Yuan Y, Zhang L. Scalable Influence Maximization in Social Networks under the Linear Threshold Model[C]// ICDM 2010, The 10th IEEE International Conference on Data Mining, Sydney, Australia, 14-17 December 2010. IEEE, 2010.

[25] Pathak, Nishith, Arindam Banerjee, and Jaideep Srivastava. "A generalized linear threshold model for multiple cascades." 2010 IEEE International Conference on Data Mining. IEEE, 2010.

[26] Zhang, Huiyuan, et al. "Recent advances in information diffusion and influence maximization in complex social networks." Opportunistic Mobile Social Networks 37.1.1 (2014): 37.
[27] He, Xinran, et al. "Influence blocking maximization in social networks under the competitive linear threshold model." Proceedings of the 2012 siam international conference on data mining. Society for Industrial and Applied Mathematics, 2012.

[28] Litou, Iouliana, et al. "Real-time and cost-effective limitation of misinformation propagation." 2016 17th IEEE International Conference on Mobile Data Management (MDM). Vol. 1. IEEE, 2016.

[29] Budak, Ceren, Divyakant Agrawal, and Amr El Abbadi. "Limiting the spread of misinformation in social networks." Proceedings of the 20th international conference on World wide web. ACM, 2011.

[30] Zang, Wenyu, et al. "Discovering multiple diffusion source nodes in social networks." Procedia Computer Science 29 (2014): 443-452.

[31] Comin, Cesar Henrique, and Luciano da Fontoura Costa. "Identifying the starting point of a spreading process in complex networks." Physical Review E 84.5 (2011): 056105 .

[32] Jain, Alankar, Vivek Borkar, and Dinesh Garg. "Fast rumor source identification via random walks." Social Network Analysis and Mining 6.1 (2016): 62.

[33] Luo, Wuqiong, Wee Peng Tay, and Mei Leng. "How to identify an infection source with limited observations." IEEE Journal of Selected Topics in Signal Processing 8.4 (2014): 586-597.

[34] Dong, Wenxiang, Wenyi Zhang, and Chee Wei Tan. "Rooting out the rumor culprit from suspects." 2013 IEEE International Symposium on Information Theory. IEEE, 2013.

[35] Zhu, Kai, and Lei Ying. "Information source detection in the SIR model: A sample-path-based approach." IEEE/ACM Transactions on Networking (TON) 24.1 (2016): 408-421 